of clinical behaviours and practice, and identifying palliative patients.

Following initial scoping the IT enabler of choice, My RightCare (MRC) was identified for the locality and was subsequently grant funded by the NHS England Nursing Technology Fund. Unlike other solutions MRC creates a patient-centric unified care plan, specifically being clinician friendly, with functionalities such as auto-population and flagging in your own system. Created care plans, are then shared (with consent) across all health care IT systems; primary and secondary care, including urgent care; ambulance and NHS 111 services. This coupled with developing a suite of tools to support GPs and provider colleagues to utilise as they choose, has supported, engaged, and empowered them to improve coordination of end of life care in the county and create a social movement on the frontline.

We would like to share the experience of a large rural county in achieving clinical engagement through bottom-up transformational change. With a small budget and little authority, but with innovative technology and a different approach we are implementing EPaCCS in our locality. And there are no project plans!

\section{P-63 IMPLEMENTATION OF A PALLIATIVE CARE ELECTRONIC PATIENT RECORD SHARED WITH GPS, DNS AND THE OUT OF HOURS SERVICE}

${ }^{1}$ Susan Salt, ${ }^{1}$ Marie Bonnis, ${ }^{1}$ Nicky Parkes, 'Debra Green, ${ }^{1}$ Carol Wylde, ${ }^{1}$ Victoria Murphy, ${ }^{2}$ Bridget Lord. 'Trinity Hospice, Blackpool, UK; ${ }^{2}$ Blackpool Teaching Hospitals NHS Foundation Trust

10.1136/bmjspcare-2016-001245.87

\section{Aims of the Project}

1. To move to an electronic patient record across all clinical departments accessible to local community services enabling access to real time information about a patient's care and condition shared across the hospice, general practice, district nursing services and out-of-hours service

2. Enable hospice staff to remotely access a patient's up-to-date record enabling advice given and care delivered to be recorded without having to find a set of case notes or returning to base

3. Reduced amount of telephone and fax traffic between primary care and the hospice around clarifying care, medication and other key factors such as known allergies

4. Saving time on administration and record keeping to enable more clinical time.

Over a period of nine months using a change management approach an instance of the electronic patient record (EMIS) was developed for each clinical team, based on a common holistic assessment. At all stages staff engagement and communication was a high priority.

Roll out was staggered over a one month period with GO LIVE starting with the smaller day time services, to iron out any teething problems, using the lessons learnt in the bigger 24-hour services including both an adult and children's in-patient unit. (For patient confidentiality reasons the bereavement service was excluded from the whole process.)

\section{Outcomes}

1. Having completed level 2 of the information governance toolkit and compliance with information governance requirements locally all healthcare professionals in the community over 24 hours are able to access real time information about all patients known to the specialist palliative care service

2. The specialist palliative care service can access the records of patients referred to them and those where advice is requested

3. Accuracy of medication histories, compliance and allergy recording has improved

4. The amount of time lost by clinical staff making phone calls has reduced by a third.

\section{P-64 THE CHALLENGES INVOLVED IN EMBEDDING THE PILOT PALLIATIVE CARE DATASET WITHIN AN ELECTRONIC PATIENT RECORD (SYSTMONE)}

Sarah Wells. Marie Curie Hospice, West Midlands, Solihull, UK

10.1136/bmjspcare-2016-001245.88

Background Our hospice was accepted as a pilot site for the Pilot Palliative Care Data set by Public Health England (PHE) in July 2015. The hospice was paper-lite using the electronic patient record system-SystmOne (S1).

Aim To embed any future dataset within S1 to ensure that data was captured within standard working practices enhance patient care as well as improve reporting of patient outcomes.

Methods There were three distinct elements to this pilot project:

1. Changing culture and the language used by staff The pilot dataset included measures of performance status (Karnofsky), complexity (Phase of illness) and patient outcome measures. Champions identified within each staff discipline and education was provided on all measures.

2. Configuration of S1 for data collection Extensive reconfiguration of S1 was required involving incorporation of the concept of "Spells of care", creation of templates and protocols

3. Creation of reporting tools

The granularity of data for the dataset required a different method of reporting to that used previously. This enabled access to a huge amount of raw data but has required an external data analyst company to produce the required reports

Results Seven months after commencing the pilot:

1. Changing the culture and language is taking time. This requires a stepwise approach but gradually these elements are becoming embedded across all sectors

2. SystmOne has now been configured to include all the data items required.

3. Reports were submitted to PHE but are now being fine-tuned to meet our needs.

Interpretation and conclusions To embed a new dataset within an EPR including all of the above elements takes longer than the six months anticipated. Although the palliative care dataset will not be taken forward, the elements of it are proving to be valuable resources that we feel will improve patient care and enable us to evidence the quality of care provided.

\section{P-65 DEVELOPING DOCUMENTATION FOR END-OF-LIFE CARE}

Joy Farquharson. St Andrew's Hospice, Airdrie, UK

10.1136/bmjspcare-2016-001245.89 
Following the recommendations of the Neuberger Report on the use of the Liverpool Care Pathway (LCP) and subsequent guidance from Scottish Government (SG), we developed a means of recording, evidencing and auditing end-of-life care which met requirements of the SG's four principles, as follows:

- Informative, timely, sensitive communication

- Significant decisions about a person's care, including diagnosing dying, are made on the basis of multi-disciplinary discussion.

- Each individual person's physical, psychological, social and spiritual needs are recognised and addressed as far as is possible.

- Consideration is given to the wellbeing of relatives or carers attending the person.

Thirteen key objectives were agreed in relation to evidencing and auditing appropriate end of life care. These were translated into an End of Life Care Record (EoLCR).

The project comprised two stages:

Stage One (5 week pilot)

- Ward A: Patients identified as being in the last days/hours of life, care documented in EoLCR

- Ward B: Patients identified as being in the last days/hours of life, care documented in Personal Care Records (PCR).

- All data compared against the 13 objectives

- Minor amendments were made and the EoLCR rolled out to all wards

Stage Two (Retrospective Audit)

A retrospective audit was undertaken of all deaths within the hospice over three months of implementation and compared against the 13 objectives.

We aimed to develop a robust means of evidencing end of life care which followed SG's 4 principles. This relates to NHS Scotland's 2020 vision that care will be provided to the highest standards of quality and safety, with the person being centre of all decisions.

The retrospective audit showed improvements in recording end of life care, please refer to Abstract P-65 Table 1 on page A34.

Using the EoLCR allowed us to evidence the principles of good end-of-life care especially in relation to nutrition, hydration and communication with patients/families.

\section{\begin{tabular}{|l|l}
\hline P-66 THE ROLE OF THE VOLUNTEER RESEARCH ASSISTANT \\
\hline
\end{tabular} IN A HOSPICE SETTING}

Charlotte Brigden, Jeff Southon, Declan Cawley. Pilgrims Hospices in East Kent, Canterbury, UK

\subsection{6/bmjspcare-2016-001245.90}

Context A hospice setting has a small, busy research team and the volunteer research assistant is an important role within the team, providing additional support for delivery of research projects and related activities.

The role was first introduced in 2014 following the development of research infrastructure and capacity building within the organisation.

What has been achieved? Examples where volunteers have supported hospice research include assisting:
- the research team with the set up and co-ordination of a clinical trial. Including support with the organisation and collation of study materials, meetings and training to the site.

- the running of a research event. A volunteer supported the set up and running of the event by helping organise attendance lists and other materials for the day, greeting and registering attendees on arrival.

- data entry into an electronic spreadsheet of study data collected on paper forms. They have also learnt some data checking skills along the way too.

What have we learned? Our experience with involving volunteers as part of the research team has been a rewarding one working towards the hospice's strategy to become increasingly research active in order to provide the best care for patients and their families. It has provided the hospice with additional research capacity to deliver projects and other research related activities.

Volunteers with different skills, expertise and experience of the organisation has meant we have been able to match their input to the needs of the research activity.

Experience of involving volunteers has meant we have been able to develop a role description for a 'volunteer research assistant'.

Introducing the role of the 'volunteer research assistant' has helped to promote ownership of this unique position to the volunteer involved. There is value in developing this role to provide regular support in developing, setup, delivering and disseminating research within hospices.

\section{P-67 HOSPICE RESEARCH LEADS AS INSTIGATORS OF RESEARCH}

${ }^{1}$ Kathy Armour, ${ }^{2}$ Anne Finucane, ${ }^{3}$ Emma Carduff, ${ }^{4}$ Lisa Graham-Wisener, ${ }^{5,6}$ Laura Green, ${ }^{7}$ Nicky Agelopolous. ${ }^{1}$ Marie Curie Hospice, Solihull, UKi ${ }^{2}$ Marie Curie Hospice, Edinburgh, UK; ${ }^{3}$ Marie Curie Hospice, Glasgow, UK; ${ }^{4}$ Marie Curie Hospice, Belfast, UK; ${ }^{5}$ University of Bradford, UK; ${ }^{6}$ Marie Curie Hospice, Bradford, UK; ${ }^{7}$ Marie Curie, London, UK

\subsection{6/bmjspcare-2016-001245.91}

From the inception of the hospice movement, research has been identified as essential to the delivery of high quality palliative and end-of-life care. Despite delivering compassionate care and excellent symptom management, research has not been embedded in hospice care. Recognising the need for greater engagement in research, our organisation first piloted the Research Lead programme in 2011. The aim was to facilitate participant involvement in palliative care studies, to facilitate evidence based practice, and to create a culture of enquiry.

Full time Research Leads were appointed at three hospices in England, Scotland and Northern Ireland. A 2013 evaluation identified the benefits of such relationship-based, proactive roles. These included increased participation of patients, staff and carers in research, and enhanced research support and mentoring to all professional groups. This positive outcome contributed to programme expansion, with our organisation now employing five hospice-based research leads, each developing collaborations with regional academic institutions.

Outcomes associated with increased investment in research staff at hospices are both tangible and intangible. Tangible outcomes include numbers of participants recruited for research studies, and traditional academic outputs: publications and research grants involving hospice staff, students supervised and conference presentations. Other outcomes, relate to impact and 